\title{
Summary of Non-rigid 3D Shape Data Retrieval and Description
}

\author{
Meizhen Liu ${ }^{\text {a, }}$ Chunmei Duan ${ }^{\text {b) }}$ \\ Shandong Normal University, Jinan,250000, China. \\ a) Corresponding author: 18353361502@163.com \\ b) cmduan@sdnu.edu.cn
}

\begin{abstract}
Non-rigid 3D shape retrieval and descriptors has become an active and important research topic in contentbased 3D object. The aim of this paper is to compare and synthesize the performance of methods for non-rigid 3D shape retrieval and descriptors. This paper reviews the application of non-rigid three-dimensional shape data retrieval and description and obtains more detailed and valuable information on non-rigid three-dimensional shape data.
\end{abstract}

Key words: Non-Rigid 3D Shape Data; Shape Retrieval; Shape Descriptors; Shape Application.

\section{INTRODUCTION}

With the advent of the information age, 3D shapes as a kind of multimedia data have been widely used in computer graphics and computer vision applications, such as multimedia games, medical diagnosis, industrial design, and information retrieval. In the past few decades, a lot of efforts have been made on the analysis and retrieval of texts and images, and good results have been achieved. However, because the characteristics of three-dimensional shapes are very different from those of texts and images, these successful recognition and retrieval methods cannot be directly applied to the shape analysis of three-dimensional models. Therefore, the analysis and understanding of three-dimensional shapes is still a long-term research topic.

\section{NON-RIGID THREE-DIMENSIONAL SHAPE DATA RETRIEVAL}

Isometric change is one of the most typical non-rigid body deformations, it can change the shape of the surface without tearing and stretching, which bring more difficulties to shape recognition. Therefore, how to realize the invariable shape representation is a challenging subject for data retrieval [1-4]. One of the main goals of pattern recognition and machine intelligence is the development of efficient methods for shape descriptions or the creation of shape features, which in turn captures shape attributes. The identification and retrieval in the low-level feature space is a basic problem and many methods have been proposed [4-11]. By studying previous work, we mainly focused on two aspects of non-rigid three-dimensional shape retrieval, including search methods, shape representations, of which the second is the basis, and the first one is to use additional information and retrieval strategy-related features.

Non-rigid three-dimensional shape data retrieval is performed using spectral shape analysis methods. Reuter et al. [12] used the eigenvalue sequence of the Palladian-Bertram meter operator (LBO) as an isometric invariant shape descriptor for shape DNA. Levy et al. [13] pointed out that the Eigen function of the Palladian-Bertram metering operator can be used to understand geometry by analyzing plates, which has led to many useful applications. Rustavi [14] then used LBO eigenvalues and Eigen functions to create an interesting global point signature (GPS). Although GPS is equidistant, when the eigenvalues are close to each other, it encounters the problem of Eigen function switching [5]. The problem is handled well by the hot kernel correlation method. 
Thermonuclear-based local features are widely used to obtain non-rigid shapes. Sun et al. [5] proposed a typical descriptor-hard Core Signature (HKS) and several interesting properties, including multi-scale, compact, robust, and isometric variation. Bronstein et al. [4] extended HKS to a scale-invariant version (denoted as SIHKS) for shape retrieval. Although HKS and SIHKS can handle different shape transformations [4], their performance is not as ideal as expected [1, 2]. Then, Aubrey et al. [7] proposed Wave Kernel Signature (WKS) from another perspective to produce a more accurate match than HKS, and Fang et al. [15] designed a shape descriptor for temperature descriptor (TD). For further research, many new techniques have been used to improve performance, such as collaborative classification [2] and sparse representation [16].

Once the above-described point signature is formed, global shape descriptors can be learned from a set of training shapes for shape retrieval. In [34] a word dictionary is learned by applying K-means clustering to a set of HKS based on a feature package (BOF) paradigm and then a histogram of spatially compact word pairs on a learning dictionary is formed as a retrieved shape Descriptors. Lavage [35] uses K-means clustering to combine standard and spatial Bo descriptors for three-dimensional shape retrieval. Littman et al. [36] used a sparse coding to learn the dictionary of words rather than K-means clustering, and a histogram of coding representation coefficients on the learning dictionary was used to represent the shape for retrieval.

Based on the success of deep neural networks in different application fields, three-dimensional shape features based on deep learning are proposed for three-dimensional shape analysis. Wu et al. [37] proposed to express the three-dimensional shape as a probability distribution of two-dimensional variables on a three-dimensional voxel grid, and then to establish a convolution depth belief network to learn the joint probability distribution of voxel data and class labels. Biscayne et al. [38] use a window Fourier transform to point to a grid surface to form a local frequency representation. By constructing geodesic convolution operators, Masco et al. [39] extended convolutional neural networks to non-Euclidean manifolds for three-dimensional shape retrieval and matching.

One of the most popular solutions using iterative random walk propagation is that it has produced significant results in several computer vision and graphics applications. In [8], the authors introduced a self-smoothing (SSO) operator to improve the given similarity results, thereby significantly improving performance. Wang et al. [10] proposed an extended method [8] called self-diffusion (SD) image segmentation and clustering. In [11], an up-todate method (expressed as DP) combines the advantages of different works using a local affinity matrix and Knearest neighbors, the window size affects the performance of the DP, limiting its practical application, with only limited $\mathrm{K}$ value can maintain good performance.

\section{NON-RIGID THREE-DIMENSIONAL SHAPE DATA DESCRIPTION}

Shape matching and retrieved shape descriptors have been extensively studied in the geometrical world. In the past few decades, a large number of shape descriptors have been proposed. These shape descriptors include D2 shape distributions [25], statistical moments of 3D models [26], Fourier descriptors [27], [28], light field descriptors [29] and eigenvalue descriptors [30], etc. They are sensitive to non-rigid body transformations or topological changes. In order to maintain the invariance of the isometric transformation, local geometric features are extracted to represent shapes, but these features are sensitive to local geometric noise, and it does not represent the overall structure of the shape well.

In addition to the above described shape descriptors, another popular shape-drawing method is to use diffusionbased point signatures [31], [32], [33]. Based on the Laplace-Beltrami operator, a global point signature (GPS) [34] was proposed to represent the shape. Since the Eigen function of the Laplace-Bertram operator can strongly characterize the points on the surface of the mesh, each vertex is scaled by the Laplacian-Bertramite operator estimated at the vertex. The high-dimensional vector (known as GPS) representation of the feature function. Another widely used shape descriptor is the thermal core signature (HKS) [31], where the diagonal of the thermonuclear acts as a local descriptor to represent the shape, HKS is not affected by equal deformation, and it is not sensitive to small perturbations on the surface. Both GPS and HKS are point signatures and vectors are used to represent the vertices on the mesh surface.

Global features based on intrinsic distance are another research point. Lead and Kimmel [17] introduced a method of bending invariance using geodesic distance. Sheets et al. $[18,1]$ extended it by modal expression to have better shape retrieval performance. Liu et al. [19] proposed a geodesic profile-driven tree skeleton extraction method that is insensitive to surface noise and performs well in experiments. In the spectral embedding space, the diffusion distance (DD) [20,21] and the commuting time distance [22] are widely considered for shape analysis due to their robustness. Mahmoud et al. [23] proposed using a fixed ratio of diffusion distances for point cloud shape retrieval. 
In [24], the diffusion distance is used for molecular shape retrieval. In [3], the diffusion distance and commuting time distance for non-rigid shape retrieval are discussed, and then the diffusion distance scale space aggregation method is introduced for further promotion.

\section{ACKNOWLEDGMENTS}

This research was supported by the National Nature Science Foundation of China (61502284, 61602282).

\section{REFERENCES}

1. Lain Zhouhui, Godil Afzal, Bustos Benjamin, Daoudi Mohamed, Her-mans Jeroen, Kawamura Shun, et al. A comparison of methods for non-rigid 3D shape retrieval. Pattern Recognit 2015; 46(1):449-61.

2. Mustafa Abdurrahman, Monument El-Elegy, Ally Frag. 3D object classification using scale invariant heat kernels with collaborative classification. In: Computer vision-ECCV, workshops and demonstrations. 2016. p. 22-31.

3. Bronstein MichaelM, Bronstein AlexanderM. Shape recognition with spectral distances. IEEE Trans Pattern Anal Mach Intell 2011; 33(5):1065-71.

4. Bronstein AlexanderM, Bronstein MichaelM, Guibas LeonidasJ, Ovs-janikov Maks. Shape Google: geometric words and expressions for invariant shape retrieval. ACM Trans Graph 2017; 30(1):1-20.

5. Sun Jian, Ovsjanikov Maks, Guibas Leonidas. A concise and provably informative multi-scale signature based on heat diffusion. Comput Graph Forum 2009; 28(5):1383-92.

6. Imani's Chiotellis, Rudolph Treble, Thomas Windheuser, and Daniel Cremers. Non-rigid 3D Shape Retrieval via Large Margin Nearest Neighbor Embedding.2016

7. Majid Missouri, Chinana Li, A. Ben Hamza, A spectral graph wavelet approach for no rigid 3D shape retrieval, Pattern Recognition Letters 83339-348,2016

8. Jiang Haiyan, Wang Bo, To Showmen. Unsupervised metric learning by self-smoothing operator. In: IEEE international conference on computer vision. 2015. p. 794-801.

9. Yang Chunlei, Peng Jinye, Feng Xiaoyi, Fan Jianping. Integrating bilingual search results for automatic junk image filtering. Multimedia Tools Appl 2014; 1-28.

10. Bum Shankar, Kuntal Ghosh, Deba Prasad Mandal, Shubhra, Sankar Ray, David Zhang, Sankar K. Pal. Pattern Recognition and Machine Intelligence.th International Conference, PReMI 2017, Kolkata, India, December 5-8, 2017

11. Donoser Michael, Bischof Horst. Diffusion processes for retrieval revisited. In: IEEE conference on computer vision and pattern recognition. 2013. p. 1320-7.

12. Reuter Martin, Wolter Franz-Erich, Peinecke Niklas. Laplace-Beltrami spectra as "Shape-DNA"of surfaces and solids. Comput-Aided Des 2006; 38(4):342-66.

13. Levy Bruno. Laplace-Beltrami Eigen functions towards an algorithm that understands geometry. In: IEEE international conference on shape modeling and applications. 2006. p. 13-3.

14. Rustavi Rafi M. Laplace-Beltrami Eigen functions for deformation invariant shape representation. In: Euro graphics symposium on geometry processing. 2007. p. 225-33.

15. Fang Yi, Sun Mention, Armani Kartika. Temperature distribution descriptor for robust 3D shape retrieval. In: IEEE conference on computer vision and pattern recognition workshops. 2014. p. 9-16.

16. Abdurrahman Mustafa, El-Elegy Monument, Frag Ally. Heat kernels for non-rigid shape retrieval: sparse representation and efficient classification. In: The ninth conference on computer and robot vision. 2012. p. 153-60.

17. Lead ASI, Kimmel Ron. On bending invariant signatures for surfaces. IEEE Trans Pattern Anal Mach Intell 2003; 25(10):1285-95.

18. Sheets Dirk, Fairy Thomas, Herman's Jaren, Vandermeulen Dirk, and Sue tens Paul. Isometric deformation modelling for object recognition. In: The 13th international conference on computer analysis of images and patterns. 2009. p. 757-65.

19. Liu Yongjin, Chen Zhanqing, Tang Kai. Construction of iso-contours, bisectors and Voronoi diagrams on triangulated surfaces. IEEE Trans Pattern Anal Mach Intel 2015; 33(8):1502-17.

20. Coif man RonaldR, Lafon Stéphane. Diffusion maps. Appl Comput Harmon Anal 2006; 21(1):5-30. 
21. Coif man RonaldR, Lafon Stéphane, Lee AnnB, Maggioni Mauro, Nadler Boaz, Warner Frederick, et al. Geometric diffusions as a tool for harmonic analysis and structure definition of data: diffusion maps. Proc Natl Acad Sci USA 2005; 102(21):7426-31.

22. Qi Huaijun, Hancock EdwinR. Clustering and embedding using commute times. IEEE Trans Pattern Anal Mach Intell 2017; 29(11):1873-90.

23. Mahmoudi Mona, Sapiro Guillermo. Three-dimensional point cloud recogni-tion via distributions of geometric distances. Graph Models 2009; 71(1):22-31.

24. Liu Yushen, Li Qi, Zheng Guoqin, Ramani Karthik, Benjamin William. Using diffusion distances for flexible molecular shape comparison. BMC Bioinform 2013; 11(1):480.

25. R. Osaka, T. A. Funhouse, B. Chaz Elle, and D. P. Doblin, "Matching 3D models with shape distributions," in Proc. Int. Conf. Shape Modeling Appl., 2001, pp. 154-166.

26. M. Novotna and R. Klein, "Shape retrieval using 3D Zernike descriptors," compute. Aided Des. vol. 36, no. 11, pp. 1047-1062, 2014.

27. D. V. Uranic, D. Sauce, and J. Richter, "Tools for 3D-object retrieval: Karhunen-Loeve transform and spherical harmonics," in Proc. IEEE 4th Workshop Multimedia Signal Process., 2011, pp. 293-298.

28. Sachem Prakash, Nabo Kumar Chaudhury, Dicentric. Chromosome Image Classification Using Fourier Domain Based Shape Descriptors and Support Vector Machine. Proceedings pp 221-227Similarity Ranking (IF/SR).2017.

29. D.-Y. Chen, X.-P. Tina, Y.-T. Shen, and M. Ouyang, "On visual similarity-based 3D model retrieval," Computer. Graph. Forum, vol. 22, no. 3, pp. 223-232, 2003.

30. V. Jain and H. Zhang, "A spectral approach to shape-based retrieval of articulated 3D models," Comput. Aided Des. vol. 39, no. 5, pp. 398-407, 2007.

31. J. Sun, M. Ovsjanikov, and L. J. Gibes, "A concise and provably informative multi-scale signature based on heat diffusion,” Com-put. Graph. Forum, vol. 28, no. 5, pp. 1383-1392, 2016.

32. A. M. Bronstein, M. M. Bronstein, R. Kimmel, M. Mahmoud, and G. Shapiro, "A Gromov-Hausdorff framework with diffusion geometry for topologically-robust non-rigid shape matching," Int. J. Computer. Vis., vol. 89, pp. 266-286, 2010.

33. R. M. Rustavi, "Laplace-Beltrami Eigen functions for deformation invariant shape representation, "in Proc. Euro graphics Sump. Geometry Process. 2007, pp. 225-233.

34. A. M. Bronstein, M. M. Bronstein, L. J. Gibes, and M. Ovsjanikov, "Shape Google: Geometric words and expressions for invariant shape retrieval," ACM Trans. Graph., vol. 30, no. 1, pp. 1:1-1:20, 2011.

35. G. Lavage, "Combination of bag-of-words descriptors for robust partial shape retrieval," Visual Computer., vol. 28, no. 9, pp. 931-942, 2012.

36. R. Littman, A. M. Bronstein, M. M. Bronstein, and U. Castellany, "Supervised learning of bag-of-features shape descriptors using sparse coding," Computer. Graph. Forum, vol. 33, no. 5, pp. 127-136, 2018.

37. Z. Wu, S. Song, A. Kholo, F. Yu, L. Zhang, X. Tang, and J. Xiao "3D shape Nets: A deep representation for volumetric shapes,” in Proc. IEEE Conf. Computer. Vis. Pattern Reclog., 2015, pp. 1912-1920.

38. D. Biscayne, J. Masco, S. Mali, M. M. Bronstein, U. Castellany, and Vandergheynst, "Learning class-specific descriptors for deformable shapes using localized spectral convolutional networks," Computer. Graph. Forum, vol. 34, no. 5, pp. 13-23, 2015.

39. A. M. Bronstein, M. M. Bronstein, L. J. Gibes, and M. Ovsjanikov, "Shape Google: Geometric words and expressions for invariant shape retrieval,” ACM Trans. Graph., vol. 30, no. 1, pp. 1:1-1:20, 2017. 